Fecha de recepción: noviembre 2018

Fecha de aceptación: marzo 2019

Versión final: julio 2019

\section{El pulgarcito educado}

Gabriela Chavez Mosquera *

Resumen: El Whatsapp como herramienta educativa. Hoy la educación universitaria se embarca en un reto, ya no hacen falta instrucciones sino ingenio para construir futuros escenarios de aprendizaje. Por tanto, el uso de aplicaciones se podría constituir en una ingeniosa herramienta. El desafío es transformar en consecuencias positivas el uso de las nuevas tecnologías y las aplicaciones a partir de la promoción de una práctica activa de la teoría para la naturalización de los conceptos. Resolviendo la interrogante de Serres en su libro Pulgarcita. ¿Qué trasmitir? ¿A quién transmitir? y ¿Cómo trasmitirlo?

Estas nuevas generaciones demandan espacios formadores donde la tecnología se convierta en un aliado, promoviendo el uso de app-capacitadoras, que incitan a buscar posibilidades nuevas y los docentes como mediadores no permitan que app-dependientes limiten los actos de los estudiantes. Como docentes, el reto es mejorar la pedagogía para dejar marcas que perduren positivamente en los estudiantes.

Palabras clave: Aplicaciones - educación - entornos de clase - espacios formadores - escenarios de aprendizaje.

[Resúmenes en inglés y portugués en las páginas 50-51]

${ }^{(*)}$ Lic. en Animación Digital, Universidad San Francisco de Quito, Ecuador. Especialista en Marketing Digital, Universidad Técnica Particular de Loja. MFA Computer Art School of Visual Arts, NY, U.S.A. Doctoranda UP.

Al observarlos, con admiración, enviar mas rápidamente de los que yo nuca podría hacerlo con mis gordos dedos, enviar (digo) SMS con los dos pulgares, los he bautizado -con la mas grande ternura que pueda expresar un abuelo- Pulgarcita y Pulgarcito. Este es su nombre, mas bonito que la vieja palabra, pseudo-cientifica, de dáctilo (Serres, Pulgarcita, p. 8).

Hoy la educación universitaria se embarca en un reto, ya no hacen falta instrucciones sino ingenio para construir futuros escenarios de aprendizaje. Es así como la tecnología no se debería convertir en el mas despiadado enemigo del docente, por el contrario, forma parte de la flexibilidad que esta generación demanda. 
Como sostiene Gardner (2014), al hablar de tecnología se inscribe tanto el hardware como el software que ha formado parte de nuestra especie desde hace miles de años. Todos los seres humanos reaccionan a los estímulos y a las acciones que la tecnología nos ofrece desde que nacemos hasta que morimos.

La tecnología se clasifica en:

- Herramientas y máquinas: es la tecnología en el sentido tradicional de la palabra (hacha, maquina de vapor) y que normalmente esta hecha de madera, metal, plástico u otros materiales disponibles.

- Información: que se puede trasmitir bien a través de nuestro propio cuerpo, bien mediante tecnologías de distinto tipo y obra del ser humano (noticias, ocio, mapas, entradas de enciclopedia).

- Información trasmitida por una maquina o una herramienta concreta (el televisor que emite noticias locales o internacionales constituye un medio de comunicación (Gardner, 2014, p. 31).

Es así donde era de tecnología, Smartphone, la información, es decir el conjunto concreto de "conocimiento" se intercambia por un medio, entendiéndose como el instrumento donde esta información se concentra y permite el procesamiento de información para una futura toma de decisiones.

En el marco de este contexto tecnológico la reflexión de Mc Luhan (1967) cobra total vigencia. Vivimos actualmente en una época para cruzar barreras y borrar viejas categorías, encontrando un equilibrio funcional entre la imaginación de enseñar con nuevas tecnologías y el aprender con nuevas herramientas.

"El medio es el mensaje" es una mirada a nuestro alrededor para ver que está sucediendo. Es una colisión-oscopio de situaciones interrelacionadas entre sí" (Mc Luhan, p. 10). Para Mc Lughan y como lo asevera Gardner (2014), las aplicaciones no serían más que un nuevo medio de comunicación. Por tanto, el uso de aplicaciones (Whatsapp) se podría constituir en una ingeniosa herramienta didáctica para la educación.

Es aquí donde surge la misma interrogante que se planteó Serres en su libro Pulgarcita. ¿Qué trasmitir? ¿A quién transmitir? y ¿Cómo trasmitirlo?

Antes de responder a dichas interrogantes cabe hacer un paréntesis y poner sobre la mesa que, ante esta hipotética situación, el Whatsapp como herramienta educativa, quien debería cambiar sus prácticas es el docente, sus creencias son el primer obstáculo a superar (Anijovich y Cappelletti, 2017).

Entendemos por "creencia" el repertorio de supuestos y respuestas rutinarias a los problemas profesionales que, generalmente, no tienen base teórica que las sustenten, pero configuran el comportamiento de los profesores. Representan una plataforma de partida relevante y critica en el desarrollo de las prácticas docentes y funcionan como el fundamento central que los guía (Anijovich y Cappelletti, 2007, p. 41).

Acercándonos entonces a las interrogantes de Serres. ¿Qué trasmitir? ¡El saber! ¿A quién transmitírselo? A esta nueva generación de universitarios y ¿Cómo trasmitirlo? Con nuevas tecnologías, aplicaciones, que promuevan el aprendizaje. Ya lo sostuvo Brunner (1966) La comunicación y el pensamiento van juntos. Es así como el reto sería que los docentes 
den el valor al Whatsapp (aplicaciones) por el valor de usarlo, por la retribución que este devuelve. Es decir, por el conocimiento.

Se debería ejecutar menos practicas de enseñanza basados en una tradición mimética, donde su principal objetivo es presentar, ejecutar, evaluar, premiar, fijar y avanzar. La educación de esta era demanda cambios fundamentales de la enseñanza, cambios que en algún momento repercutirán en el futuro. Ahora educar se convierte más que en una responsabilidad en una iniciativa pedagógica. La enseñanza poderosa, termino que lo sostiene Maggio (2012) es crear una propuesta original que transforme. Donde la resolución de problemas complejos pueda ser abordados de manera original con estudiantes entusiasmados y comprometidos. Construir diálogos que iluminan (Maggio, 2014).

Estas nuevas generaciones demandan espacios formadores donde la tecnología se convierta en un aliado, promoviendo el uso de app-capacitadoras, que incitan a buscar posibilidades nuevas y los docentes como mediadores no permitan que app-dependientes limiten o determinen los actos de los estudiantes (Gardner, 2014).

Necesitamos de una tradición trasformadora donde el docente tenga un compromiso profundo hacia el estudiante y su aprendizaje, basado en lo experimental acercándose así a una educación constructivista y no conductista. Apoyando a lo que sostiene Schon (1992) la meta de hoy en día es formar profesionales más reflexivos.

En base a lo expuesto el Diseño Grafico se convertiría en una buena coartada para poner en práctica el enunciado que sostiene este articulo. "El diseño es un campo complejo que tiene en la proyectualidad y la comunicación los ejes constitutivos de su hacer" (Mazzeo, 2017, p. 19). Por eso la propuesta del uso de whatsapp en los entornos de clase se aplica a una práctica de reflexión en la acción donde la teoría no es dictada como tal, pero en el accionar hay ejecuciones teóricas ofreciendo "argumentaciones visuales que intervienen en ese complejo montaje en el que los interlocutores interactúan” (Mazzeo, 2017, p. 28). Entonces, resulta una propuesta novedosa que el docente por medio de un mensaje grupal envíe cada mañana una imagen / texto para analizar; y, aplicando las estrategias de didáctica de corrección de proyectos se segmentaria de cierta forma la didáctica. La imagen o texto serviría para que el alumno indague sobre las intensiones de lo enviado (COMPRENDER), proceso que lo realizaría de manera autónoma una vez visualizada la imagen, y una vez que la recepción de la imagen es comprobada, el docente (CHEQUEA) contenidos teóricos en la practica, afianzando así conceptos. Finalmente, para la hora del encuentro personal (ACLARA Y ACENTUA) la asimilación del aprendizaje por medio de aplicaciones reiterativas.

Como lo sostiene Gardner (2014), para algunos jóvenes, esos mensajes tecleados son incluso mas íntimos que una conversación directa. Por lo tanto, con la intimidad que los masajes de texto permiten, el alumno indirectamente estaría realizando una actividad autónoma que incentive a la reflexión en la aplicación de conceptos y procedimientos haciendo que se convierta en un conocimiento inerte.

Como docentes, tenemos un reto de cada día cuestionarnos como mejorar la pedagogía y dejar marcas que perduren positivamente en los estudiantes. Es un reto al que no todos están dispuestos por que pueden fracasar o acertar en sus intentos. Pero al menos se actúa sobre el problema, se innova sobre la marcha. "En el sentido de la enseñanza poderosa que se formula en tiempo presente, ser original es también no copiarse a si mismo, reconocer cada realidad para construir allí pedagogía” (Maggio, 2012, p. 26). 
Por último, no se puede concluir este articulo reflexivo sin cuestionar qué profesionales queremos formar. En los ambientes de aula el celular y el uso de aplicaciones se debe convertir en un aliado y el docente debe permitir que los estudiantes puedan reflexionar mientras estén en contacto con dichas aplicaciones.

Todos los medios nos dan una paliza de pies a cabeza. Son tan penetrantes en sus consecuencias personales, políticas, económicas, estéticas, psicológicas, morales éticas y sociales que no dejan parte sin tocar, afectar o modificar. El medio es el mensaje. Es imposible comprender el cambio social y cultura si no se conoce el modo en que funcionan los medios como entornos (Mc Luhan, 1967, p. 26)

El desafío es transformar en consecuencias positivas el uso de las nuevas tecnologías y las aplicaciones a partir de la promoción de una práctica activa de la teoría para la naturalización de los conceptos. Ya lo dijo Litwin (1997), en lo que ella denominó el metaanálisis de la clase, definido como la construcción del conocimiento a partir de la reconstrucción de lo recientemente vivido en un plano diferente (como se cita en Maggio, 2012). Pensar en una educación que inspire por medio de creaciones colectivas.

\title{
Bibliografía
}

Anijovich, R. y Cappelletti, G. (2017). La evaluación como oportunidad. Buenos Aires: Paidós. Gardner, H. y Davis, K. (2014). La generación APP: Cómo los jóvenes gestionan su identidad, su provacidad y su imaginación en el mundo digital. Argentina: Paidós.

Hernandez Pina, M. (sin año). Las relaciones entre pensamiento y lenguaje según Piaget, Vygotsky, Luria y Bruner. Recuperado de: https://digitum.um.es/jspui/bitstream/10201 /21940/1/05\%20Las\%20relaciones\%20entre\%20pensamiento\%20segun\%20Piaget\%20 Vygotsky.pdf

Maggio, M. (2012). Enriquecer la enseñanza: Los ambientes con alta disposición tecnológica como oportunidad. Buenos Aires: Editorial Paidós.

Maggio, M. (TED Talks). (2014, marzo,06). Si la enseñanza: Mariana Maggio at TEDxUCES. Recuperado de: https://www.youtube.com/watch?v=zE5eH52Vqkw

Mazzeo, C. (2017). Diseño y sistema: bajo la punta del iceberg. Buenos Aires: Ediciones Infinito.

McLuhan, M. y Fiore, Q. (1967). El medio es el mensaje: un inventario de efectos. Buenos Aires: La Marca Editora.

Schön, D. (1992). La formación de profesionales reflexivos. Barcelona; Editorial Paidós

Serres, M. (2012). Pulgarcita. Paris: Manifiestos le Pommier.

\begin{abstract}
The Whatsapp as an educational tool. Today the university education embarks on a challenge since you don't need instructions but ingenuity to build future scenarios for learning. Therefore, the use of applications could be a nifty tool. The challenge is to transform in positive consequences the use of new technologies and applications from the promotion of an active practice of the theory for the naturalization of the concepts.
\end{abstract}


Resolving the question of Serres in his book Thumbelina. What transmit? Who do you transmit? And how do I transmit it? These new generations demand trainers where spaces technology becomes an ally, promoting the use of app-trainers, which incite to search for new possibilities and teachers as mediators do not allow app-dependent limit the acts of students. As teachers, the challenge is to improve the pedagogy to leave marks that last positively in students.

Keywords: Applications - Education - classroom environments - spaces trainers - learning scenarios.

Resumo: O WHATSAPP como uma ferramenta educacional. Hoje, o ensino universitário embarca em um desafio desde que você não precisa de instruções mas a engenhosidade para construir cenários futuros para a aprendizagem. Portanto, o uso de aplicações pode ser uma ótima ferramenta. O desafio é transformar em consequências positivas para a utilização de novas tecnologias e aplicações a partir da promoção de uma prática activa da teoria para a naturalização dos conceitos. Resolver a questão de Serres, em seu livro Thumbelina. O que transmitir? Que você transmitir? E como posso transmiti-lo?

Estas novas gerações formadores procura espaços onde a tecnologia se torna um aliado, promovendo o uso do app-formadores, que fomentam a busca de novas possibilidades e professores como mediadores não permitem a app-limite dependente dos actos dos estudantes. Como professores, o desafio é melhorar a pedagogia para deixar marcas que duram positivamente em estudantes.

Palavras chave: Aplicações - Educação - ambientes de sala de aula - espaços formadores cenários de aprendizagem.

[Las traducciones de los abstracts fueron supervisadas por el autor de cada artículo] 